- Oscar Beijbom at the University of California, Berkeley, and the software can zip through Catlin's gigantic photo album - currently around one million photographs - in a matter of months.

The software is just one example of how coral researchers are embracing advances in computer science and software to speed up under-sea mapping of reefs around the world. Combined with high-quality imagery and sensors that collect standardized biological data about reefs, these tools could unleash an era of semi-automated data collection and monitoring, freeing up ecologists to spend less time processing data and more time doing research.

"It's a tremendous step forward," says Mark Eakin, who manages the Coral Reef Watch programme for the US National Oceanic and Atmospheric Administration (NOAA) in College Park, Maryland. "When you aren't limited by the speed of people going through and manually processing images, the yield of information is just so much greater."

\section{OCEAN OF DATA}

Coral researchers' entry into the world of big data comes none too soon. Long limited by the size of their diving fins and the capacity of their oxygen tanks, marine ecologists are racing to expand their surveys to document and understand the longer-term impacts of rising ocean temperatures and acidification. The bleaching of corals around the world that has accompanied the epic 2015-16 El Niño warming event in the tropical Pacific Ocean has only heightened concerns.

González-Rivero's goal is to cover as much territory as possible to get a sense of how different corals and reefs are responding to these stresses. Computers will never replace the human eye, nor will they obviate the need for detailed underwater investigations and laboratory research, but they can speed up the basic surveys, he says. "What we are trying to do is find a compromise where we get enough information to understand the reef, but at a much faster pace and in a much cheaper way."

The quality does not have to be compromised: according to Beijbom's unpublished results, the deep-learning system agrees with the human eye on features in coral photos about $81 \%$ of the time - impressive considering that even two experts are likely to agree only $84 \%$ of the time.

Beijbom plans to launch the algorithm in a few months' time for anyone submitting pictures to his website CoralNet, which already uses computer-assisted systems to help the automated analysis of images. The service is free thanks to funding from NOAA, and 420 users from a variety of institutions, including NOAA, have already uploaded nearly 269,000 images to the site. The best results seem to come from use of a semi-automated program in which the computer does simple analyses and alerts human experts to cases that

it's not confident about, Beijbom says.

In many ways, González-Rivero says, marine science is catching up with the terrestrial sciences, which have been developing tools to gather and process copious amounts of data from satellites and aircraft for decades. The software and hardware can't be directly translated to analysing seas, however: the ocean swallows light, so it is difficult to study anything but the shallowest

\section{"We need} an army of people making high-quality measurements." reefs from above.

That has pushed coral researchers to adapt the tools. At Michigan State University in East Lansing, for example, biophysicists David Kramer and Atsuko Kanazawa have modified a handheld sensor originally designed for agricultural research.

When used on land, the sensor measures information such as fluorescence in plants, the carbon content of the soil, the temperature of the air and the humidity. Around 300 sensors are in use in 18 countries, and every time a researcher or a government official takes a reading, the data are uploaded to a central server for analysis.

The modified system, dubbed CoralspeQ, pings reefs with different kinds of light and records the returning spectral signal in 256 wavelengths, from ultraviolet to infrared. These data can be used to measure a reef's photosynthetic activity, for instance, by measuring the fluorescence of chlorophyll in symbiotic algae that provide their host corals with oxygen and nutrients. Knowing how much photosynthetic activity is taking place, and where, could help researchers to identify stressed systems, Kramer says.

The devices use commercially available sensors and are built with the help of 3D printers. Kramer and Kanazawa hope to bring down the cost of the underwater version from its current US\$500 and get it into the hands of as many scientists as possible. "We need an army of people making high-quality measurements," Kramer says.

\section{COMPUTER-ASSISTED VISION}

Marine microbiologist Arjun Chennu has developed an underwater imaging system to collect even more detailed data across a greater radiation spectrum. Coral ecologists then annotate the images, and the information is fed into a neural-network algorithm that is based on open-source machine-learning software and is similar to the one developed by Beijbom. The machine's 'hyperspectrum' means that it can capture much more information than can the human eye, says Chennu, who works at the Max Planck Institute for Marine Microbiology in Bremen, Germany. This makes it easier to differentiate between corals that look similar in standard images. "For example, we resolve the often-used 'other coral' categories into their proper taxonomic types, and also include sponges, macroalgae and seagrass in our predictions," he says.

Others have adapted commercially available software that is already used to map landscapes and analyse landslides by overlapping 2D images into 3D models. PhD student John Burns at the University of Hawaii at Manoa's Institute of Marine Biology uses a program called Agisoft PhotoScan, which costs \$549 for an educational licence for the professional edition. Free software is available, but it is less sophisticated, Burns says.

The models - which can achieve a resolution of just 1 millimetre when used with good cameras - can be analysed by people or computers to identify coral species and quantify reef coverage. But, because they're 3D, they can also be used to track structural changes as reefs bleach and break down owing to high ocean temperatures - a new kind of ecological information.

For Burns, the beauty of the method is its simplicity: data can be collected quickly and with minimal training. "This method just lets you take hundreds of thousands of single-lens images with your camera, and then you are essentially stitching them together," he says.

\section{STANDARDIZED STORE}

Sophisticated technologies aren't the only answer, says Emily Darling, a marine ecologist with the Wildlife Conservation Society in New York City. Because separate research efforts are collecting ever-greater quantities of data on coral reefs, it is important that they collect standardized data sets and store them in a repository that can be accessed by the entire community.

In an effort to collect systematic data on the recent global bleaching event, for example, Darling and her colleagues came up with a very simple technology - an Excel spreadsheet that scientists around the world can use to register various data on reef conditions. The value is that when scientists come out of the water, they can immediately import and analyse their data, and Darling now has uniform results from more than 61,000 reef colonies in 13 countries. Roughly $58 \%$ showed bleaching.

Ultimately, Darling says, coral ecologists need to converge on some kind of a central repository for the full suite of information that they are collecting around the world. "We need places where data are accessible, where they are telling stories, and where people can go and figure out whether conservation actions are working or not," she says. "We need to be able to answer those questions a lot faster."

\section{CORRECTION}

The story 'The paper promoters' (Nature 536, 113-114; 2016) should have made clear that Altmetric.com collects data from both mainstream and social media. 